\title{
Recent Work on Culture and Schizophrenia: Epidemiological and Anthropological Approaches
}

\author{
Simon Dein* \\ Queen Mary University of London, Centre for Psychiatry, UK
}

Submission: February 11, 2017; Published: June 07, 2017

*Corresponding author:Simon Dein, Queen Mary University of London, Centre for Psychiatry, Old Anatomy Building, Charterhouse Square, London, UK, Email: s.dein@ucl.ac.uk

\section{Introduction}

Schizophrenia is observed worldwide in diverse cultures. However as Abed \& Abbas [1] note, the supposed universality of the incidence and prevalence of schizophrenia has been seriously challenged. It is now widely accepted that the life-time prevalence and incidence of this disorder vary considerably in time and place. A lower, $50 \%$ concordance between identical twins suggests that environmental or stochastic influences play a significant role in its causation [2]. Here I discuss the role of culture on presentation, attributions and outcomes citing studies by both psychiatric epidemiologists and anthropologists. Transcultural psychiatrists have often argued that schizophrenia is universal: it has similar manifestations in all cultures. This view is consistent with the patho plastic model which is dominant in Western psychiatry and which asserts that schizophrenia is fundamentally similar across cultural groups, with differences expressed only in the content (e.g. demons, government conspiracy) of symptoms, not in terms of the underlying cause and structure [3,4]. As I shall discuss below schizophrenia may take different forms in different. The question rises as to whether cross cultural findings suggest that schizophrenia is a discreet diagnostic entity? This is not a systematic review; rather I have deliberately chosen studies that clearly indicate cultural influences on schizophrenia and contribute to the debate on its diagnostic status.

\section{Culture and Hallucinations}

Culture shapes hallucinations in terms of identification, experience, content, form frequency, meaning, distress they elicit, and in the way in which others respond [5]. One of the most significant ways in which culture affects the recognition of the experience of hallucination rests on the understanding of reality in the culture in question. Different cultural models of reality may lead to differing levels of reporting and thus the kinds and rates of hallucinatory experience may vary between cultures in epidemiological studies due to different theories of the world and not just differing levels of experience.

Bauer et al. [6] compared people with schizophrenia in 7 different countries (Austria, Poland, Lithuania, Georgia, Pakistan, Nigeria, and Ghana). In all settings, patients were more likely to report auditory than visual hallucinations, but the 1-year prevalence rates ranged considerably: auditory hallucinations from 67\% (Austria) to 91\% (Ghana) and visual from $4 \%$ (Pakistan) to $54 \%$ (Ghana).

Anthropologists and psychiatrists have found that to some extent, the hallucinations associated with serious psychotic disorder are "pathoplastic," meaning that they are shaped by local cultural expectation and meaning. Rural Africans are more likely to hallucinate about ancestor worship; Christians are more likely to experience hallucinations about Christ, Mary, and Satan. The content of hallucinations may change over time. Mitchell \& Vierkant [7] compared hallucinations in patients admitted to an East Texas hospital during the 1930s with those reported in patients in the same hospital in the 1980s (patients were matched for age, race, and gender distribution). Their findings indicated that hallucinations of the 1930s reflected the desire for material goods linked to the Great Depression while those of the 1980s reflected the new technological tools of the 1980s. More significantly, the command hallucinations of the 1930s were primarily benign and religious ("live right", "lean on the Lord"), but those of the 1980s were negative and highly destructive ("kill yourself", "kill your mother"). The authors suggested that the more negative commands of the later period derived from a more negative and hostile environment.

Suhail \& Cochrane (2002) examined Pakistanis living in Britain (BP; N=53), Pakistanis in Pakistan ( $\mathrm{PP} ; \mathrm{N}=98$ ) and British White (BW; $\mathrm{N}=50$ ). The content of the patients' delusions and hallucinations were obtained from medical records and 
key-workers' reports from All-Saints Hospital, Birmingham and two psychiatric units in Lahore. Any reference to delusions and hallucinations was coded in accordance with the classification scheme developed for the Present State Examination. These authors observed that the most dissimilar pair was the white British patients and the Pakistani patients living in Pakistan. More specifically, the British patients were significantly more likely (compared to the Pakistani patients) to hear, for instance, voices commenting on behavior, personality, and actions; commands to kill self or others; and voices calling bad names. The Pakistani participants more frequently claimed to experience criticising, threatening, or insulting voices.

Kent \& Wahass (1996) compared the auditory hallucination of patients with schizophrenia in Saudi Arabia and the United Kingdom. Saudi Arabian patients were more likely to experience hallucinations with religious content, while the British were more likely to report a running commentary.

Luhrmann et al. [8] interviewed 60 adults diagnosed with schizophrenia- 20 each in San Mateo, California; Accra, Ghana; and Chennai, India. Overall, there were 31 women and 29 men with an average age of 34. Participants were asked how many voices they heard, how often, their attributions relating to the auditory hallucinations, and what their voices were like. While many of the African and Indian subjects registered predominantly positive experiences with their voices, in contrast, not one American did. The U.S. participants were more likely to experience their voices as violent and hateful. Thus the authors conclude that hallucinations associated with schizophrenia or serious psychotic disorder may be less caustic, on average, for persons in the non-West, compared to those in the West. Luhrmann et al. [8] postulates that people who develop serious psychotic disorder pay selective attention to a constant stream of many different auditory and quasi-auditory events because of different "cultural invitations"-variations in ways of thinking about minds, persons, spirits and so forth. She labels this the 'social kindling' hypothesis- if persons with psychosis experience more benign hallucinations in some cultural settings than in others, it may render the voice-hearing experience will be less clinically harmful.

Finally in relation to content, Mosotho, Louw, Calitz (2011) in South Africa found that the core symptoms of schizophrenia among Sesotho speakers do not differ significantly from other cultures. However, the content of psychological symptoms such as delusions and hallucinations is strongly affected by cultural variables. Somatic symptoms such as headaches, palpitations, dizziness and excessive sweating were prevalent among the Sesotho-speaking participants suffering from schizophrenia.

\section{The Expression of Schizophrenia Cross Culturally and schizophrenic Subtypes}

Mclean (2015) notes that schizophreniais generally treated by psychiatrists as a single (continuous) disorder diagnosed according to reliable, internationally-accepted criteria. There is disagreement regarding the optimal representation of schizophrenia's structure:

I. a single (continuous) entity, with clinical variation represented as dimensions within a single class, or

II. Two or more distinct, separate entities, with variation indicative of multiple classes grouped under the label "schizophrenia" [9-11]. Cross cultural studies may facilitate clarification of this question.

Studies suggest there is significant variation in the expression of schizophrenia across ethnically diverse populations. In a cross cultural study Mclean et al. (2014) compared both lifetime frequencies of DSM-IV criterion A (the core symptom criterion of the internationally recognized DSM classification system) symptoms and types/content of delusions and hallucinations in schizophrenia populations from Australia $(n=776)$, India $(n=504)$ and Sarawak, Malaysia $(n=259)$, to assess clinical heterogeneity. Differences were found in both criterion. A symptom composition and in symptom content. Indian individuals suffering with schizophrenia endorsed negative symptoms more frequently than other sites, whereas individuals from Sarawak reported disorganized symptoms more frequently. Delusions of control and thought broadcast, insertion, or withdrawal were found less frequently in Sarawak than in Australia. Of interest a subgroup of 20 Indian individuals with schizophrenia reported no lifetime delusions or hallucinations. These findings question the widely held view in cultural psychiatry that schizophrenia is fundamentally similar across cultural groups, with differences in only the content of psychotic symptoms, but equivalence in structural form.

Relatedly, Barrett (2004) found that his attempt to translate the PresentState Examination from English into the Iban language failed when assessing thought insertion and withdrawal. In the Iban culture, thinking is held to arise from the heart-liver region. It is not contained in the mind, which is somehow contained in the brain-a more Western conception. The Iban do not have a cultural conception of thought insertion or manipulation, even in interactions between spirits and human beings. Barrett (2004) questions whether this derives from different cultural conceptions of 'thinking' and 'feeling' and the different ways these cultural conceptions shape the schizophrenic individuals experiences?

\section{Attributions of Psychotic Illness}

Cultures differ in their attributions of mental illness. Understanding attribution is important for clinicians since it relates to pathways to care (eg religious vs biomedical) and may impact medication compliance and treatment outcome In Eastern societies mental disorders are frequently attributed to spiritual or supernatural reasons: fate, evil spirits, punishment by an ancestor. Today belief in jinn is deeply embedded in many Islamic societies. Jinn are created by Allah but are invisible 
and have a lifecycle similar to humans. Lim, Hoek \& Blom [12] argue that Islamic patients who experience hallucinations often attribute them to jinn spirits. They found 105 articles about jinn and their relationship with mental disorders, including 47 case reports. About 66 percent of those reports included a biomedical diagnosis. About half of the cases involved a person with schizophrenia or a related disorder, while the rest of the patients had mood disorders, epilepsy or obsessive-compulsive disorder. Moreover, findings from several case reports suggested that the attribution of psychiatric symptoms to jinn also affects the treatment and course of patients' mental disorders. Attribution of psychosis to spirit possession in Buddhist groups Vietnam has also been described by Gaines [13].

Kate et al. [14] examined the prevalence of supernatural beliefs among patients with schizophrenia attending an outpatient clinic in Chandigarh, India. The study demonstrated that super-natural beliefs were quite common in north Indian community with the majority of the patients attributing their symptoms to one of the supernatural causes. Sixty two percent of patients admitted that people in their community maintained beliefs in sorcery and other magico-religious phenomena. One fourth to half of patients believed in ghosts/evil spirit (26\%), spirit intrusion (28.8\%) and sorcery (46.6\%). Two-third patients held that mental illness could be caused by sorcery, ghosts/evil spirit, spirit intrusion, divine wrath, planetary/astrological influences, dissatisfied or evil spirits and bad deeds of the past. Forty percent of the subjects attributed mental disorders to more than one of these causes. About half of the patients (46.6\%) maintained that only performance of prayers was sufficient to improve their mental status. Few patients $(9.6 \%)$ asserted that magico-religious rituals were sufficient to improve their mental illness but about one-fourth $(24.7 \%)$ admitted that during recent episode either they or their caregivers had performed diverse magico-religious rituals.

With an interest in the post secular, Jones et al. [15] conducted an in-depth qualitative analysis of 19 interviews of individuals diagnosed with psychotic disorders in community mental health and service user advocacy settings in the USA All subjects reported some childhood exposure to religion but none were fundamentalist or orthodox. The authors found that this syncretic process affected not only the content of psychotic experiences-what delusions or hallucinations are about-but also the type of arguments or logics used to justify particular interpretations. The majority of participants mentioned ongoing and self-conscious struggles to demarcate their experiences as the products of the real world or a "crazy" mind. With equal frequency, participants weighed up and debated competing secular and supernatural explanations, often juxtaposing and blending different explanatory frameworks. Subjects with schizophrenia often invoked both scientific and religious explanations to make sense of their psychosis and expressed self -conscious tensions between the secular and non-secular. All expressed clinical concerns about the reality of their experiences and also secular uncertainty as to the status of supernatural or magical explanations. There was a complex bricolage of explanatory accounts.

\section{The Outcome of Schizophrenia}

Schizophrenia is generally assumed to have a better prognosis in non-industrialized societies. The course of the disease was considered be generally more severe in industrialized nations than in developing countries. Those individuals diagnosed with schizophrenia in "developing" countries (e.g., Mauritius, Colombia, Nigeria, and India) have been found to experience more short- and long-term recovery outcomes than people in Europe and the US [16-20]. As Hopper [18] notes, there is a "relatively constant ... odds of recovery ratio of roughly 1.5 favoring the non-industrial group."

The evidence most often cited derives from three World Health Organization (WHO) cross-national studies. The first two studies were the International Pilot Study of Schizophrenia (IPSS, 1967) and the Determinants of Outcome of Severe Mental Disorders (DOSMeD, 1978). It was found that patients with schizophrenia living in 'developing countries had a much more favorable prognosis and higher rates of remission than patients living in so-called "developed" countries like the United States and those in Western Europe.

Hopper \& Wanderling [21] examined the validity and robustness of the original WHO studies' findings after 13 years asking whether there were any confounding variables that could have accounted for the difference which are unrelated to the effects of culture. They examined 809 people in 13 research centres and 11 countries, many of whom were the same people from the original WHO studies. Additionally they also examined a separate sample from Hong Kong and Madras. Their findings strongly confirmed the original findings of the WHO study and they found that the potential sources of bias were not strong enough to justify the great difference in the course and prognosis of the disease in developing countries.

How do we understand these differences in prognosis cross culturally? The first explanation pertains to the fact that the patients in the developing countries include people who do not really have schizophrenia- there is little support for this hypothesis. Second, various cultural factors have been invoked. In India it is postulated that the family remain fully involved in the treatment, unlike in America; in India patients often live in joint families, where they may function as useful family members without having to function as primary breadwinners or primary caretakers; entry- level work may be less stressful and less demanding than it is in America, where many such jobs are in fast-paced, high people-contact settings like McDonalds; fewer families in India exhibit high expressed emotion than in America- a well -documented risk factor for relapse in schizophrenia; psychotic hallucinations may seem more similar 
to standard religious practice than they do in America; in India, there may be a different understanding of self-coherence, there may be lesser degrees of stigma.

Luhrman [19] proposes that "social defeat"-an actual social encounter in which one person physically or symbolically loses to another one- may be one of the social factors that may impact illness experience and uses original ethnographic research to argue that social defeat is a common feature of the social context in which many people diagnosed with schizophrenia in America live today. Finally Jenkins [22] suggests that less high expressed emotion in developing countries might improve prognosis.

There have been some criticisms of these findings. Although a number of socio-cultural factors have been considered as contributing to variation in the course of schizophrenia in different settings, little evidence exists from low-income countries that clearly demonstrates the beneficial influence of these variables. Patel et al. [20] suggest that the finding of better outcomes in developing countries necessitates re-examination for five reasons: significant methodological limitations of the World Health Organization studies; the lack of evidence pertaining to the specific socio-cultural factors which apparently contribute to the better outcomes; increasing anecdotal evidence describing the abuse of basic human rights of people with schizophrenia in developing countries; novel evidence from cohorts in developing countries illustrating much gloomier picture than originally believed; and, rapid social and economic changes are undermining family care systems for people with schizophrenia in developing countries.

These authors contend that the results of the WHO studies were artifactual. Mortality rates for those with schizophrenia in developing countries are very high with many of them attributed to suicide. Also, the inpatient psychiatric care in many developing countries can be extremely poor and resemble torture more than healthcare. They document conditions of severe abuse and neglect which have been documented in India and many countries in East and South Asia. Overall, they argue that the evidence suggests a very negative view of mental illness in developing countries

These authors argue that the study of the long-term course of this mental disorder in developing countries is a major research question and believe it is time to thoroughly and systematically explore cross-cultural variation in the course and outcome of schizophrenia.

\section{Conclusion}

The evidence cited suggests that different cultures display markedly different symptoms and manifestations of schizophrenia, often due to the cultural norms and sociocentricity of a culture. There is substantial research indicating that basic schizophrenia symptoms such as hallucinations, an hedonia, antisocial behavior, depressive symptoms, emotional processing, and mood induction, vary across cultures [6,23-25].
How might differences in folk psychology impact the experience of schizophrenia? The concepts of sociocentricity, individualism, and collectivism are critical to understanding the cultural variance in schizophrenia. There is data suggesting that the values of a society-collectivistic or individualistic-could affect emotion processing and mood induction in those suffering from schizophrenia [24] and thus impact presentation and outcome. Second there may be cultural differences in perceptual processes. Westerners tend to pay more attention to salient objects in an image rather than the background and tend to have a perceptual (concrete) orientation in vision [6]. Meanwhile, East Asians pay more attention to the background and have a more conceptual (abstract) orientation in vision [6]. These different emphases in visual and attentional processing could be the source of the differentiation in hallucination types observed among different cultures.

Finally how might cultural factors interact with biological factors in the causation of schizophrenia? There is evidence in schizophrenia of a dysfunctional HPA axis, hippocampus, and dopaminergic system. On account of these neurological differences prodromal individuals are more vulnerable to stress $[26,27]$. Cultural factors might act as stressors building on biological vulnerability to stress.

\section{References}

1. Abed RT, Abbas MJ (2014) Can the new epidemiology of schizophrenia help elucidate its causation? Irish Journalof Psychological Medicine 31: 1-5.

2. MacDonald AW, Schulz SC (2009) What We Know: Findings That Every Theory of Schizophrenia Should Explain. Schizophrenia Bulletin 35(3): 493-508.

3. Kleinman Arthur (1988) Rethinking Psychiatry: From Cultural Category to Personal Experience. The Free Press; New York, USA.

4. Kulhara Parman, Chakrabarti Subho (2001) Culture and schizophrenia and other psychotic disorders. The Psychiatric clinics of North America 24(3): 449-464.

5. Frank Larøi, Tanya Marie Luhrmann, Vaughan Bell, William A Christian Jr, Smita Deshpande, et al. (2014) Culture and Hallucinations: Overview and Future Directions, Schizophr Bull 40: S213-S220.

6. Bauer SM, Schanda H, Karakula H, Olajossy-Hilkesberger L, Rudaleviciene P, et al. (2011) Culture and the prevalence of hallucinations in schizophrenia. Comprehensive Psychiatry 52: 319325 .

7. Mitchell J, Vierkant AD (1989) Delusions and hallucinations as a reflection of thesubcultural milieu among psychotic patients of the 1930s and 1980s. Journal of Psychology 123(3): 269-274.

8. Luhrmann Padmavati Tharoor Osei (2015) Differences in voicehearing associated with psychosis in Accra, Chennai and San Mateo. Br J Psychiatry 206 (1): 41-44.

9. Kendler Kenneth S, Karkowski Laura M, Walsh Dermot (1998) The structure of psychosis: latent class analysis of probands from the Roscommon Family Study. Archives of General Psychiatry 55(6): 492499.

10. Fiedorowicz Jess G, Epping Eric A, Flaum Michael (2008) Toward defining schizophrenia as a more useful clinical concept. Current Psychiatry Reports 10(4): 344-351. 
11. Linscott Richard J, Lenzenweger Mark F, van Os Jim (2009)Continua or classes? Vexed questions on the latent structure of schizophrenia. In: Gattaz Wagner F, Busatto Geraldo (Eds.), Advances in Schizophrenia Research. Springer Science + Business Media; New York, USA, pp. 333355

12. Lim HW, Hoek, JD (2015) BlomThe attribution of psychotic symptoms to jinn in Islamic patients Transcult. Psychiatry 52 (1): 18-32.

13. Gaines Rebecca (2014) Culture \& Schizophrenia: How the Manifestation of Schizophrenia Symptoms in Hue Reflects Vietnamese Culture. Independent Study Project (ISP) Collection, pp. 1-33.

14. Kate N, Grover S, Kulhara P, Nehra R (2012) Supernatural beliefs, aetiological models and help seeking behaviour in patients with schizophrenia. Ind Psychiatry J 21(1): 49-54.

15. Jones N, Kelly T, Shattell M (2016) God in the brain: experiencing psychosis in the postsecular United States. Transcult Psychiatry 53(4): 488-505.

16. Bhugra D (2006) Severe Mental Illness Across Cultures. Acta Psychiatrica Scandanivica 113(Suppl 429): 17-23.

17. Cohen Alex (2008) Questioning an Axiom: Better Prognosis for Schizophrenia in the Developing World? Schizophrenia 34(2): 229244

18. Hopper Kim (2008) Outcomes Elsewhere: Course of Psychosis in 'Other Cultures' In: Morgan C, McKenzie K, Fearon P (Eds.), Society and Psychosis. Cambridge: Cambridge University Press, UK, pp. 198-216.

19. Luhrmann TM (2007) Social Defeat and the Culture of Chronicity: Or, Why Schizophrenia Does so Well Over There and so Badly Here. Culture, Medicine and Psychiatry 31(2): 135-172.
20. Patel Vikram, Alex Cohen, Rangaswamy Thara, Oye Gureje, et al. (2006) Is the Outcome of Schizophrenia Really Better in Developing Countries? Revista Brasiliera Psiquiatria 28(2): 129-152.

21. Hopper K, Wanderling J (2000) Revisiting the developed versus developing country distinction in course and outcome in schizophrenia: results from ISoS, the WHO collaborative followup project. Schizophr Bull 26(4): 835-846.

22. Jenkins JH (1991) Anthropology, expressed emotion and schizophrenia Ethos 19: 387- 431.

23. Brekke JS, Barrio C (1997) Cross-Ethnic Symptom Differences in Schizophrenia: The Influence of Culture and Minority Status. Schizophrenia Bulletin 23(2): 305-316

24. Habel U, Gur RC, Mandal MK, Salloum JB, Gur RE, et al. (2000) Emotional processing in schizophrenia across cultures: standardized measures of discrimination and experience. Schizophrenia Research 42(1): 57-66.

25. Bae SW, Brekke JS (2002) Characteristics of Korean-Americans With Schizophrenia: A Cross-Ethnic Comparison With African Americans, Latinos, and Euro-Americans. Schizophr Bull 28(4): 703-717.

26. Corcoran C, Walker E, Huot R, Mittal V, Tessner K, et al. (2003) The stress cascade and schizophrenia: etiology and onset. Schizophr Bull 29(4): 671-692.

27.Wilbanks, Kimberly L (2010) Schizophrenia Across Cultures: Significant Interactions of Biology and Environment Research Papers, p. 123.

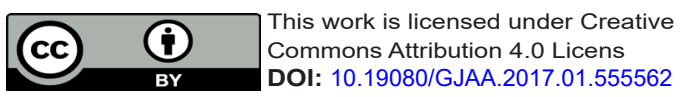


